\title{
The role of magnetic anisotropy in the Kondo effect
}

\author{
ALEXANDER F. OTTE 1,2 , MARKUS TERNES ${ }^{1}$, KIRSTEN VON BERGMANN ${ }^{1,3}$, SEBASTIAN LOTH ${ }^{1,4}$, \\ HARALD BRUNE ${ }^{1,5}$, CHRISTOPHER P. LUTZ ${ }^{1}$, CYRUS F. HIRJIBEHEDIN ${ }^{1,6}$ AND ANDREAS J. HEINRICH ${ }^{1 *}$ \\ ${ }^{1}$ IBM Research Division, Almaden Research Center, San Jose, California 95120, USA \\ ${ }^{2}$ Kamerlingh Onnes Laboratorium, Universiteit Leiden, 2300 RA Leiden, The Netherlands \\ ${ }^{3}$ Institute of Applied Physics, University of Hamburg, Jungiusstr. 11, 20355 Hamburg, Germany \\ ${ }^{4}$ Department of Physics, University of California at Berkeley, Berkeley, California 94720, USA \\ ${ }^{5}$ Institut de Physique des Nanostructures, Ecole Polytechnique Fédérale de Lausanne, CH-1015 Lausanne, Switzerland \\ ${ }^{6}$ London Centre for Nanotechnology, Department of Physics and Astronomy, Department of Chemistry, University College London, London WC1H OAH, UK \\ *e-mail: heinrich@almaden.ibm.com
}

In the Kondo effect, a localized magnetic moment is screened by forming a correlated electron system with the surrounding conduction electrons of a non-magnetic host ${ }^{1}$. Spin $S=1 / 2$ Kondo systems have been investigated extensively in theory and experiments, but magnetic atoms often have a larger spin ${ }^{2}$. Larger spins are subject to the influence of magnetocrystalline anisotropy, which describes the dependence of the magnetic moment's energy on the orientation of the spin relative to its surrounding atomic environment ${ }^{3,4}$. Here we demonstrate the decisive role of magnetic anisotropy in the physics of Kondo screening. A scanning tunnelling microscope is used to simultaneously determine the magnitude of the spin, the magnetic anisotropy and the Kondo properties of individual magnetic atoms on a surface. We find that a Kondo resonance emerges for large-spin atoms only when the magnetic anisotropy creates degenerate ground-state levels that are connected by the spin flip of a screening electron. The magnetic anisotropy also determines how the Kondo resonance evolves in a magnetic field: the resonance peak splits at rates that are strongly direction dependent. These rates are well described by the energies of the underlying unscreened spin states.

A low density of magnetic impurities in a non-magnetic host metal can have dramatic effects on the magnetic, thermodynamic and electrical properties of the material owing to the Kondo effect, a many-body interaction between the metal's conduction electrons and the electron spin of the localized magnetic impurity ${ }^{5}$. This interaction gives rise to a narrow, pronounced peak in the density of states close to the Fermi energy ${ }^{1}$. The past decade has seen a surge of interest in the Kondo screening of individual atomic spins, as a result of experimental advances in probing individual magnetic atoms by using scanning tunnelling microscopes ${ }^{6,7}$ and single-molecule transistors ${ }^{8,9}$. When magnetic atoms are placed on metal surfaces the Kondo interaction between the localized spin and the conduction electrons is very strong, leading to high Kondo temperatures $T_{\mathrm{K}}$, in the range from 40 to $200 \mathrm{~K}$ (ref. 10). In order to probe the Kondo physics it is desirable to reduce this interaction so that the Kondo screening competes on an equal footing with external influences such as magnetic fields. It was shown recently that this can be achieved by incorporating a decoupling layer between the atomic spin and the screening conduction electrons ${ }^{11}$.

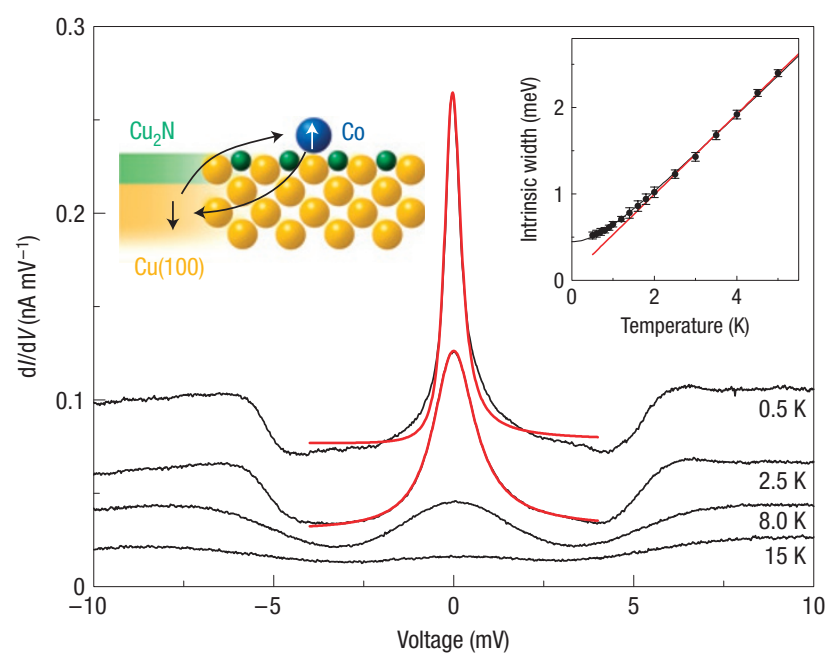

Figure 1 Temperature dependence of the Kondo resonance of a Co atom. The left inset shows a schematic drawing of a single $\mathrm{Co}$ atom bound on top of a $\mathrm{Cu}$ atom of the $\mathrm{Cu}_{2} \mathrm{~N}$ surface. Through the $\mathrm{Cu}_{2} \mathrm{~N}$ layer the $\mathrm{Co}$ atom is coupled to the electron sea of the bulk copper, which can Kondo screen the localized spin on the atom. The main panel shows differential-conductance $(\mathrm{d} / / \mathrm{d} V)$ spectra measured with the tip positioned over a $\mathrm{Co}$ atom at $0.5 \mathrm{~K}$ and higher temperatures. For each spectrum the tip height was adjusted to give a $10 \mathrm{M} \Omega$ tunnel junction $(V=10 \mathrm{mV}, I=1 \mathrm{nA})$. The red curves show fits to Fano functions broadened by the temperature of the probing tip (Fano lineshape parameter $q=17 \pm 2$ ). The right inset shows the intrinsic full-width at half-maximum of the peak as a function of the sample temperature $T$. The error bars account for uncertainties in the temperature of the tip (see the Methods section). The solid black line shows a best fit to the function $\left[\left(\alpha k_{\mathrm{B}} T\right)^{2}+\left(2 k_{\mathrm{B}} T_{\mathrm{K}}\right)^{2}\right]^{1 / 2}$, which describes the intrinsic width of the Kondo resonance in a Fermi-liquid mode $\left.\right|^{17}$. For $T \gg T_{\mathrm{K}}$ the width approaches linear behaviour with a slope $\alpha=5.4 \pm 0.1$ (red line).

In the current study, individual $\mathrm{Co}$ and $\mathrm{Ti}$ atoms are separated from a $\mathrm{Cu}(100)$ crystal by a monolayer of copper nitride $\left(\mathrm{Cu}_{2} \mathrm{~N}\right)$ (ref. 12) as sketched in the left inset of Fig. 1. We used a home-built ultrahigh-vacuum scanning tunnelling microscope with $0.5 \mathrm{~K}$ 

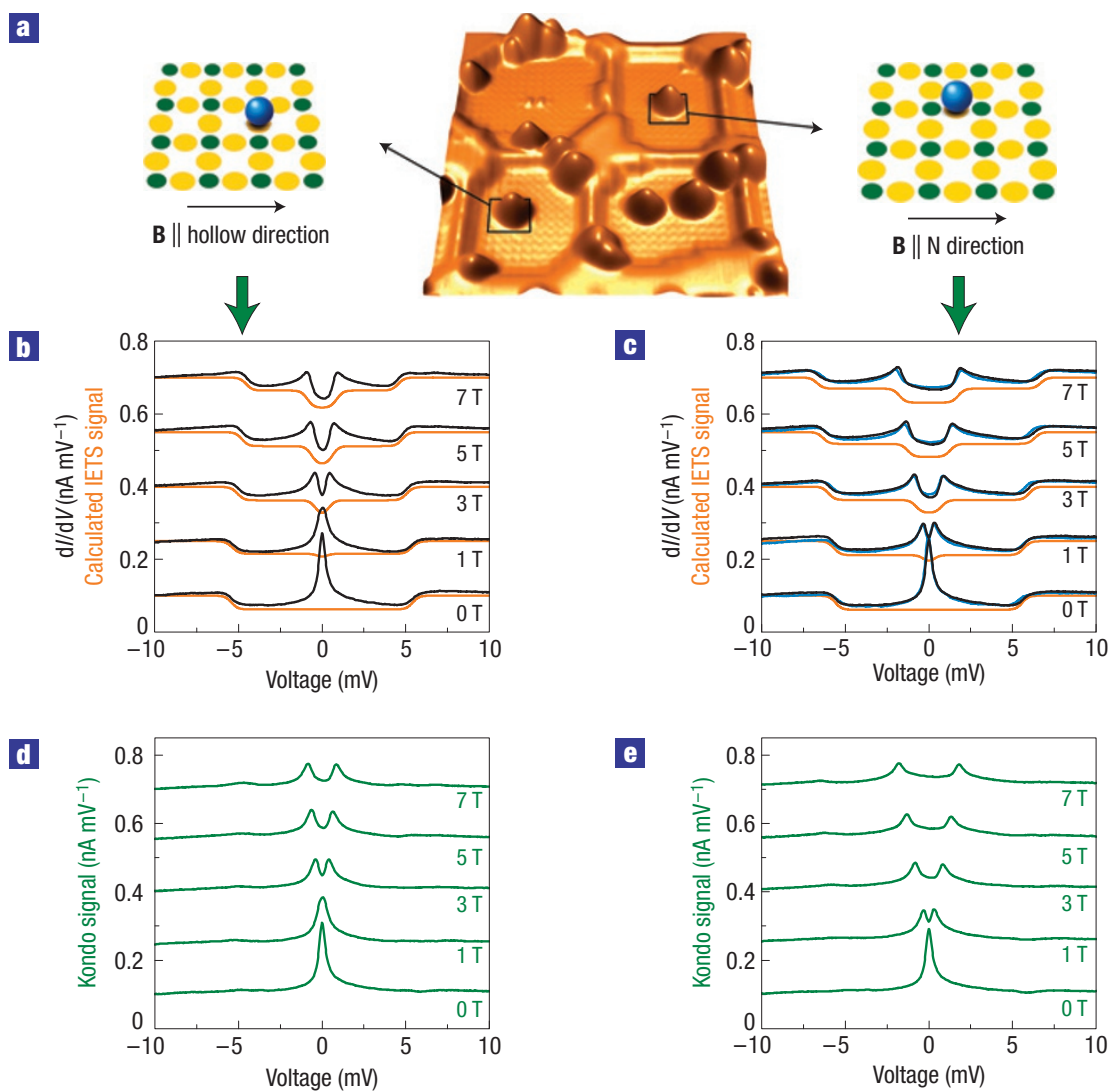

e

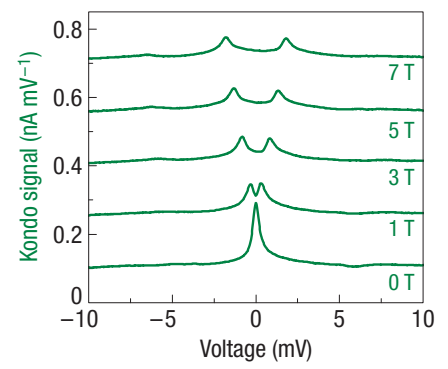

Figure 2 Anisotropic field dependence of the Kondo resonance. a, Topographic scanning tunnelling microscope image $(10 \mathrm{~nm} \times 10 \mathrm{~nm}, 10 \mathrm{mV}, 1 \mathrm{nA})$ of four $\mathrm{Cu} \mathrm{H}_{2} \mathrm{~N}$ islands with single $\mathrm{Co}$ atoms. The two diagrams at the sides sketch the adsorption site of the two marked $\mathrm{Co}$ atoms (Cu and $\mathrm{N}$ atoms are depicted as yellow and green circles, respectively). Labels indicate the two non-equivalent in-plane directions, referring to neighbouring $\mathrm{N}$ atoms or hollow sites along the direction of the magnetic field. b,c, Black curves: differential-conductance spectra taken with the tip positioned over the two Co atoms of a when a field up to $7 \mathrm{~T}$ was applied in the designated directions. Successive spectra are offset by $0.15 \mathrm{nA} \mathrm{mV}^{-1}$ for clarity. The blue curves in $\mathbf{c}$ show similar measurements on a Co atom where the magnetic field was directed perpendicular to the surface. All spectra were recorded at $T=0.5 \mathrm{~K}$. Orange curves: calculated inelastic tunnelling spectra based on the parameters obtained by fitting the positions of the outer conductance steps to equation (1). d, e, Result of subtracting the orange curves from the black curves in $\mathbf{b}$ and $\mathbf{c}$ respectively, showing the change in the spectra due to Kondo interactions.

base temperature and magnetic fields up to $7 \mathrm{~T}$ to explore this system (see the Methods section). We probed the local electronic excitations by measuring the differential conductance $\mathrm{d} I / \mathrm{d} V$, where $I$ is the tunnelling current and $V$ is the sample voltage.

A single $\mathrm{Co}$ atom bound to the $\mathrm{Cu}_{2} \mathrm{~N}$ surface shows a sharp zero-voltage peak in its conductance spectrum (Fig. 1). This peak is due to an increase in the density of states near the Fermi level that results from Kondo screening ${ }^{1}$. Such a Kondo peak has been observed in quantum dots ${ }^{13,14}$ and in atoms or molecules on surfaces ${ }^{6,7,15,16}$. The peak is well described by a thermally broadened Fano lineshape ${ }^{17}$ that results from interference between tunnelling into the Kondo resonance and tunnelling directly into the substrate ${ }^{6,7}$. For Co on $\mathrm{Cu}_{2} \mathrm{~N}$ the Fano lineshape is nearly Lorentzian, indicating that tunnelling into the Kondo resonance dominates over tunnelling directly into the substrate, presumably because the decoupling $\mathrm{Cu}_{2} \mathrm{~N}$ layer inhibits the tunnelling to the substrate.

To confirm that the conductance peak is due to a Kondo resonance, we measured the spectra at higher temperatures (Fig. 1) and observed a rapid reduction of the Kondo peak height. These spectra are broadened owing to the Fermi-Dirac distribution of the tunnelling electrons in the tip, but the intrinsic temperature behaviour of the Kondo system can be determined by carrying out a deconvolution of the measured spectra ${ }^{17}$ to remove the effect of the finite tip temperature (see the Methods section). As expected for the Kondo effect ${ }^{14}$, the intrinsic width of the resonance grows linearly with temperature at higher temperatures, but saturates at a finite value at low temperature (Fig. 1, right inset). The half-width at half-maximum $\Gamma$ of the zero-temperature peak defines the Kondo temperature $T_{\mathrm{K}}=\Gamma / k_{\mathrm{B}}$, where $k_{\mathrm{B}}$ is Boltzmann's constant. We find a Kondo temperature for Co on $\mathrm{Cu}_{2} \mathrm{~N}$ of $T_{\mathrm{K}}=2.6 \pm 0.2 \mathrm{~K}$.

To determine the effect of magnetic anisotropy on the Kondo screening, we applied a magnetic field $\mathbf{B}$ along each of the three high-symmetry directions of the sample (Fig. 2b,c). We find that the Kondo resonance splits into a double peak as in previous studies of other Kondo systems $s^{8,9,11,13,14,18,19}$. In contrast to previous studies, we observed that the peak splits at a rate that depends strongly on the direction of the field: when $\mathbf{B}$ is oriented along the direction in which the binding site is neighboured by two hollow sites (the 'hollow direction'), the splitting is much less than for the perpendicular in-plane ' $\mathrm{N}$ direction' (Fig. 2a). The strong directional dependence of the Kondo peak splitting suggests the presence of large magnetocrystalline anisotropy ${ }^{3,4}$. With $\mathbf{B}$ oriented out of plane the spectra are essentially identical to the spectra for the $\mathrm{N}$ direction. We note that this is true even though there is no symmetry between these two axes. 

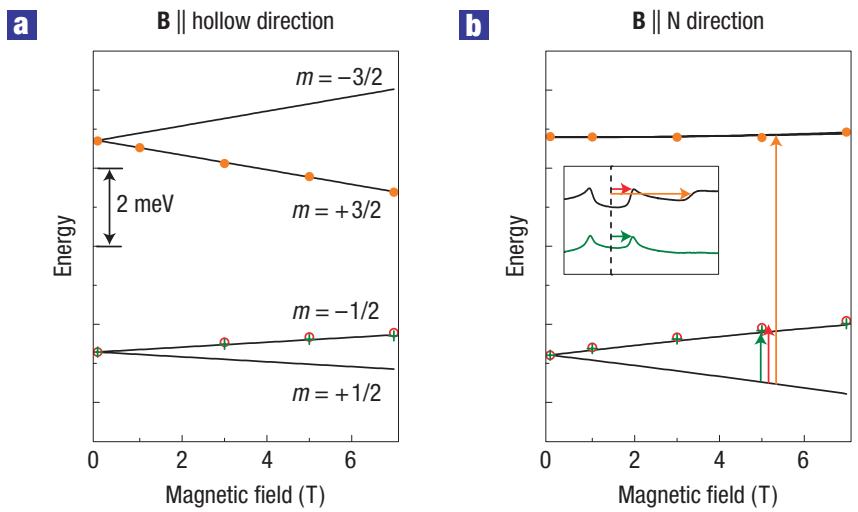

Figure 3 Energy eigenlevels for different field directions. a, Solid lines show the calculated energy levels based on equation (1) with magnetic field $\mathbf{B}$ parallel to the hollow direction (see the Methods section). Full and open circles indicate the energies of the steps and the peaks, respectively, of the spectra shown in Fig. $2 \mathrm{~b}$. The positions of the peaks in Fig. $2 \mathrm{~d}$ are represented by crosses. Values are plotted relative to the calculated ground state as illustrated by the arrows in $\mathbf{b}$. $\mathbf{b}$, The same as a but with $\mathbf{B}$ parallel to the $\mathrm{N}$ direction and data taken from Fig. $2 \mathrm{c}, \mathrm{e}$.

We are able to determine the magnitude and the magnetic anisotropy of the local spin by detecting inelastic spin excitations caused by the tunnelling electrons. In addition to the Kondo resonance peak, the spectra show an 'outer' step in the conductance near $\pm 5 \mathrm{meV}$ (Figs 1,2b,c). This step can be explained within the framework of inelastic electron tunnelling spectroscopy (IETS) as the onset of an inelastic spin excitation, which opens a new conductance channel ${ }^{11}$. The evolution of the step energy as a function of the magnitude and direction of an external magnetic field (Fig. 2b,c) can be used to quantify the magnetic anisotropy and the spin of the Co atom ${ }^{20}$. Since results obtained for $\mathbf{B}$ oriented in the $\mathrm{N}$ and out-of-plane directions are indistinguishable, we model the system as having uniaxial anisotropy along the hollow direction, which will be designated as the $z$ axis in the following. We describe the single-particle anisotropic spin behaviour-ignoring the many-body Kondo properties - with a spin Hamiltonian that is the sum of the Zeeman energy and the anisotropy energy ${ }^{4}$ :

$$
\hat{H}=-g \mu_{\mathrm{B}} \mathbf{B} \cdot \hat{\mathbf{S}}+D \hat{S}_{z}^{2},
$$

where $g$ is the Landé $g$ factor and $\mu_{\mathrm{B}}$ the Bohr magneton. The longitudinal anisotropy parameter $D$ partly breaks the zero-field degeneracy of the eigenlevels; different magnitudes of $m$, the eigenvalue of the $z$-projection $\hat{S}_{z}$ of the spin vector $\hat{\mathbf{S}}$, lead to different energies (Fig. 3). We note that this anisotropy has no influence on a system that has $S=1 / 2$.

We find good agreement between the measured outer steps and the corresponding excitation of this spin Hamiltonian (that is, the second-lowest excitation) by using $S=3 / 2$. The fit then yields $g=2.19 \pm 0.09$, which lies close to the free-electron $g$ value of 2.00 , and $D=2.75 \pm 0.05 \mathrm{meV}$, similar to the large magnetic anisotropy seen in previous studies of individual magnetic atoms on surfaces ${ }^{3,20}$. The uncertainties quoted are mainly due to slight variations in the atoms' local environments, possibly due to position-dependent strain in the $\mathrm{Cu}_{2} \mathrm{~N}$ islands ${ }^{21}$. We note that $S=5 / 2$ and higher half-integer values of $S$ also give an adequate description of the measured spectra. However, all the conclusions drawn in this work about the Kondo and low-energy spin-excitation behaviour would remain unchanged. When an atom is adsorbed on a surface the spin is generally unchanged or reduced from the free-atom value. Since the spin of a free Co atom is $3 / 2$, the spin of Co on this surface is probably $3 / 2$.

The sign of the anisotropy parameter $D$ determines whether states with large or small $|m|$ form the ground state. The positive $D$ observed here yields hard-axis (easy-plane) anisotropy, in which the two $m= \pm 1 / 2$ states have the lowest energy and are degenerate in the absence of $\mathbf{B}$. We note that even in the case of finite transverse anisotropy the twofold zero-field degeneracy of these states would not be broken, owing to Kramers' theorem. The observed outer step, used to obtain the fitting results discussed above, thus corresponds to $m= \pm 1 / 2 \rightarrow \pm 3 / 2$ transitions when $\mathbf{B}$ is applied along the $z$ direction; see Fig. 3 .

We calculated the inelastic tunnelling spectra in the absence of Kondo screening (orange curves in Fig. 2b,c). The transition energies were derived by diagonalizing the spin Hamiltonian, equation (1), using the parameter values found above. For finite B a low-energy spin excitation becomes possible as $m=+1 / 2$ and $-1 / 2$ split in energy. In addition, we used a scattering intensity operator that describes spin scattering by tunnelling electrons ${ }^{20}$ to model the relative step heights of the inner and outer transitions.

We show the effect of Kondo screening on the spectra by subtracting the calculated inelastic tunnelling spectra from the measured spectra to provide a measure of the shapes and positions of the split Kondo peak. This procedure results in symmetrically shaped peaks on a nearly flat background (Fig. 2d,e). Here we have treated the conductance as the sum of an IETS conductance channel and a separate Kondo channel in a manner similar to ref. 22. We stress that the underlying many-body physics may be more complex than the sum of these two channels ${ }^{23,24}$. We also note that the single-particle model of the inelastic tunnelling channel ignores any interference in the elastic channel due to the presence of the inelastic channel ${ }^{25}$.

The position of the split Kondo peak coincides with the calculated low-energy transition $m=+1 / 2 \rightarrow-1 / 2$ of the non-Kondo Hamiltonian of equation (1) for all measured fields and field orientations (Fig. 3). Here it makes no substantial difference whether we use the positions of the peaks in the original measurements (open circles in Fig. 3) or those that remain after subtracting the calculated IETS curves (crosses in Fig. 3). It is worth highlighting that the spin shows direction-dependent splitting of the Kondo peak only because the spin $(S=3 / 2)$ is greater than $1 / 2$. In the case of an $S=1 / 2$ impurity such anisotropic behaviour due to crystal-field effects is expected to be absent. We note that the precise nature of the splitting of the Kondo peak, especially for magnetic fields that are small compared to the Kondo temperature, is still under theoretical debate $e^{23,24,26}$.

In contrast to the hard-axis anisotropy, which we find to be the case for $\mathrm{Co}$ on $\mathrm{Cu}_{2} \mathrm{~N}$, easy-axis anisotropy $(D<0)$ would favour the $m= \pm 3 / 2$ doublet as the ground state of an $S=3 / 2$ impurity. In this situation Kondo screening is inhibited, as it would require $\Delta m=3$ transitions to be achieved through electron scattering. A Kondo resonance can be formed only if the anisotropy creates a degenerate ground state with levels connected by $\Delta m=1$ transitions (that is, the flipping of a conduction electron spin). This picture agrees well with previously studied atomic spins, Fe $(S=2)$ and $\mathrm{Mn}(S=5 / 2)$ on $\mathrm{Cu}_{2} \mathrm{~N}$, that showed no Kondo effect. Each of these was found to have easy-axis anisotropy ${ }^{20}$ and therefore does not have a ground-state doublet that is linked via $\Delta m=1$ spin excitations. Similar mechanisms, where crystalline anisotropy is responsible for creating a Kondo system from a large spin (that is, $S>1 / 2$ ), have been suggested for bulk impurities ${ }^{27,28}$. Recent theoretical investigations have shown that a Kondo effect can also occur in systems with easy-axis magnetic anisotropy, exemplified by single-molecule magnets, if an additional strong transverse 


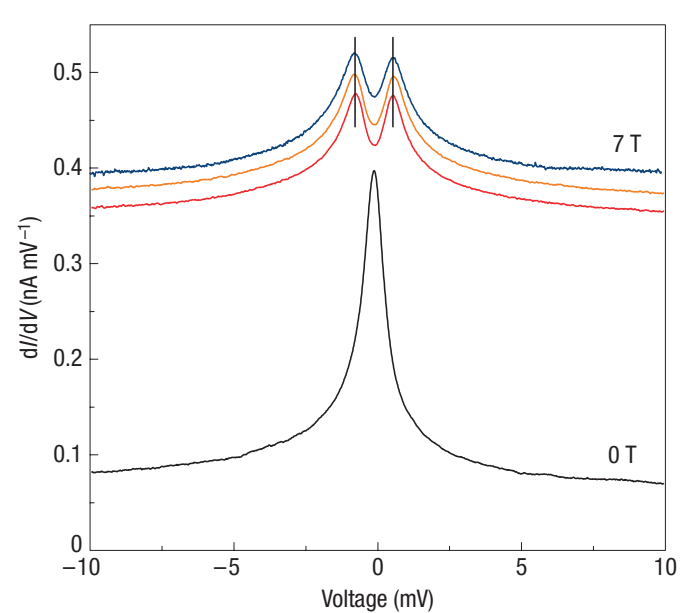

Figure 4 Kondo effect of a Ti atom. Differential-conductance spectra of individual $\mathrm{Ti}$ atoms on $\mathrm{Cu}_{2} \mathrm{~N}$ in the absence of a magnetic field (black curve) and with an external field of $7 \mathrm{~T}$ applied along the two in-plane directions (red and orange curves, offset by 0.28 and $0.30 \mathrm{nA} \mathrm{mV}^{-1}$ respectively) and oriented out of plane (blue curve, offset by $0.32 \mathrm{nA} \mathrm{mV}^{-1}$ ). Note that the $7 \mathrm{~T}$ spectra are essentially identical. All curves were measured at $T=0.5 \mathrm{~K}$.

anisotropy sufficiently mixes states with different $m$ values to create a degenerate ground state that is linked via $\Delta m=1$ transitions $^{29}$.

Unlike the measurements on Co, spectra taken on individual $\mathrm{Ti}$ atoms (Fig. 4) show a clear Kondo peak but no conductance steps due to additional spin excitations. Consequently, $\mathrm{Ti}$ on $\mathrm{Cu}_{2} \mathrm{~N}$ can be modelled as an $S=1 / 2$ system. We note that a free $\mathrm{Ti}$ atom has $S=1$ in the $3 d^{2}$ configuration, so in this case the binding to the surface presumably changes the atom's spin. Ti binds at the same location as $\mathrm{Co}$ on $\mathrm{Cu}_{2} \mathrm{~N}$, so comparable magnetic anisotropy may be expected. As shown in Fig. 4, finite magnetic fields split the Kondo peak, but no direction-dependent splitting is observed. This observation confirms that this type of crystal field can affect the Kondo resonance only of an impurity that has a spin larger than $1 / 2$.

Our result will be applicable to the Kondo effect in other systems with large spin as well, such as magnetic atoms placed directly on a metal substrate or single-molecule magnets with transverse magnetic anisotropy ${ }^{29}$. Further studies may also reveal a more quantitative understanding of the link between anisotropy and the strength of the Kondo screening. In addition, the ability to tune the Kondo effect by varying the magnitude and orientation of the magnetic anisotropy would create a new class of Kondo systems in which the screening could be manipulated directly through control of the local environment.

\section{METHODS}

Details of the experimental set-up can be found in ref. 11. Co and Ti atoms were deposited onto the $\mathrm{Cu}_{2} \mathrm{~N}$ surface at low temperature by thermal evaporation from a metal target. These atoms were subsequently placed on specific binding sites by means of vertical atom manipulation. The differential conductance $\mathrm{d} I / \mathrm{d} V$ was measured using lock-in detection with a $50 \mu \mathrm{V}$ r.m.s. modulation at $745 \mathrm{~Hz}$.

The tip was verified to have a flat density of states in the energy ranges presented, by observing essentially constant conductance spectra when the tip is placed over the bare $\mathrm{Cu}_{2} \mathrm{~N}$ and over the bare $\mathrm{Cu}$ surfaces. We determined that presence of the tip does not influence the Kondo system, but merely probes its density of states, by observing that the spectrum does not change when the junction resistance is increased.
The temperature of the sample $T_{\text {sample }}$ was regulated using a heater, whereas the tip was cooled via strong thermal contact directly to the ${ }^{3} \mathrm{He}$ refrigerator. Different refrigerator operating modes, each corresponding to a particular tip temperature $T_{\text {tip }}$, were used for different sample temperature ranges. For the deconvolution of the spectra measured when $T_{\text {sample }}<1.4 \mathrm{~K}$ we used $T_{\text {tip }}=0.5 \mathrm{~K}$, and for $1.4 \mathrm{~K}<T_{\text {sample }}<5.0 \mathrm{~K}$ we used $T_{\text {tip }}=1.8 \mathrm{~K}$. With this assignment of tip temperatures, which agree to within $0.3 \mathrm{~K}$ with the values expected for the corresponding operating modes, the intrinsic peak width was found to increase without a discontinuity at $T_{\text {sample }}=1.4 \mathrm{~K}$. The error bars were determined by carrying out deconvolution with values for $T_{\text {tip }} 0.3 \mathrm{~K}$ above and below the assigned values.

The precise parameter values found for the Co atoms of Fig. 2 are $g=2.16$ and $D=2.71 \mathrm{meV}$ for the atom with $\mathbf{B}$ along the hollow direction, and $g=2.22$ and $D=2.79 \mathrm{meV}$ for the atom with $\mathbf{B}$ along the $\mathrm{N}$ direction. These values were used to calculate the energy levels for the corresponding spins in Fig. 3 as well as the inelastic tunnelling spectra in Fig. $2 \mathrm{~b}, \mathrm{c}$.

Received 24 June 2008; accepted 13 August 2008; published 21 September 2008.

References

1. Hewson, A. C. The Kondo Problem to Heavy Fermions (Cambridge Univ. Press, Cambridge, 1997).

2. Owen, J., Browne, M. E., Arp, V. \& Kip, A. F. Electron-spin resonance and magnetic-susceptibility measurements on dilute alloys of $\mathrm{Mn}$ in $\mathrm{Cu}, \mathrm{Ag}$ and Mg. J. Phys. Chem. Solids 2, 85 (1957)

3. Gambardella, P. et al. Giant magnetic anisotropy of single cobalt atoms and nanoparticles. Science 300, 1130-1133 (2003)

4. Gatteschi, D., Sessoli, R. \& Villain, J. Molecular Nanomagnets (Oxford Univ. Press, Oxford, 2006)

5. Kondo, J. Resistance minimum in dilute magnetic alloys. Prog. Theor. Phys. 32, 37-49 (1964).

6. Madhavan, V., Chen, W., Jamneala, T., Crommie, M. F. \& Wingreen, N. S. Tunneling into a single magnetic atom: Spectroscopic evidence of the Kondo resonance. Science 280, 567-569 (1998).

Li, J., Schneider, W.-D., Berndt, R. \& Delley, B. Kondo scattering observed at a single magnetic impurity. Phys. Rev. Lett. 80, 2893-2896 (1998).

8. Park, J. et al. Coulomb blockade and the Kondo effect in single-atom transistors. Nature 417, 722-725 (2002).

9. Liang, W., Shores, M. P., Bockrath, M., Long, J. R. \& Park, H. Kondo resonance in a single-molecule transistor. Nature 417, 725-729 (2002).

10. Wahl, P. et al. Kondo temperature of magnetic impurities at surfaces. Phys. Rev. Lett. 93 , 176603 (2004).

11. Heinrich, A. J., Gupta, J. A., Lutz, C. P. \& Eigler, D. M. Single-atom spin-flip spectroscopy. Science 306, 466-469 (2004)

12. Leibsle, F. M., Dhesi, S. S., Barrett, S. D. \& Robinson, A. W. STM observations of Cu(100)-c $(2 \times 2) \mathrm{N}$ surfaces: Evidence for attractive interactions and an incommensurate $c(2 \times 2)$ structure. Surf. Sci. 317, 309-320 (1994)

13. Goldhaber-Gordon, D. et al. Kondo effect in a single-electron transistor. Nature 391, 156-159 (1998).

14. Cronenwett, S. M., Oosterkamp, T. H. \& Kouwenhoven, L. P. A tunable Kondo effect in quantum dots. Science 281, 540-544 (1998).

15. Zhao, A. et al. Controlling the Kondo effect of an adsorbed magnetic ion through its chemical bonding. Science 309, 1542-1544 (2005).

16. Wahl, P. et al. Kondo effect of molecular complexes at surfaces: Ligand control of the local spin coupling. Phys. Rev. Lett. 95, 166601 (2005).

17. Nagaoka, K., Jamneala, T., Grobis, M. \& Crommie, M. F. Temperature dependence of a single Kondo impurity. Phys. Rev. Lett. 88, 077205 (2002)

18. Shen, L. Y. L. \& Rowell, J. M. Zero-bias tunneling anomalies-temperature, voltage, and magnetic field dependence. Phys. Rev. 165, 566-577 (1968).

19. Kogan, A. et al. Measurements of Kondo and spin splitting in single-electron transistors. Phys. Rev. Lett. 93, 166602 (2004)

20. Hirjibehedin, C. F. et al. Large magnetic anisotropy of a single atomic spin embedded in a surface molecular network. Science 317, 1199-1203 (2007).

21. Choi, T., Ruggiero, C. D. \& Gupta, J. A. Incommensurability and atomic structure of $\mathrm{c}(2 \times 2) \mathrm{N} / \mathrm{Cu}(100)$ : A scanning tunneling microscopy study. Phys. Rev. B 78, 035430 (2008).

22. Appelbaum, J. A. Exchange model of zero-bias tunneling anomalies. Phys. Rev. 154, 633-643 (1967)

23. Costi, T. A. Kondo effect in a magnetic field and the magnetoresistivity of Kondo alloys. Phys. Rev. Lett. 85, 1504-1507 (2000).

24. Moore, J. E. \& Wen, X.-G. Anomalous magnetic splitting of the Kondo resonance. Phys. Rev. Lett. 85, $1722-1725(2000)$

25. Lorente, N. Mode excitation induced by the scanning tunnelling microscope. Appl. Phys. A 78, 799-806 (2004)

26. Grobis, M., Rau, I. G., Potok, R. M. \& Goldhaber-Gordon, D. in Handbook of Magnetism and Advanced Magnetic Materials (eds Kronmüller, H. \& Parkin, S.) (Wiley, New York, 2007).

27. Schlottmann, P. Effects of crystal fields on the ground state of a Ce atom. Phys. Rev. B $\mathbf{3 0}$ 1454-1457 (1984)

28. Újsághy, O., Zawadowski, A. \& Gyorffy, B. L. Spin-orbit-induced magnetic anisotropy for impurities in metallic samples of reduced dimensions: Finite size dependence in the Kondo effect. Phys. Rev. Lett. 76, 2378-2381 (1996).

29. Romeike, C., Wegewijs, M. R., Hofstetter, W. \& Schoeller, H. Quantum-tunneling-induced Kondo effect in single molecular magnets. Phys. Rev. Lett. 96, 196601 (2006).

\section{Acknowledgements}

We thank M. F. Crommie, D. M. Eigler, A. C. Hewson, B. A. Jones, J. E. Moore and J. M. van Ruitenbeek for discussions and B. J. Melior for technical assistance. A.F.O. acknowledges support from the Leiden University Fund; M.T. from the Swiss National Science Foundation; K.v.B. from the German Science Foundation (DFG Forschungsstipendium); S.L. from the Alexander von Humboldt Foundation; C.F.H. from the Engineering and Physical Sciences Research Council (EPSRC) Science and Innovation Award and M.T., C.P.L. and A.J.H. from the Office of Naval Research. H.B. acknowledges EPFL for supporting his sabbatical stay with IBM.

\section{Author information}

Reprints and permissions information is available online at http://npg.nature.com/reprintsandpermissions. Correspondence and requests for materials should be addressed to A.J.H. 